\title{
DEM Modelling of Particle Movement During Seepage Failure
}

\section{Lin Zhong}

Faculty of Construction and Environment, The Hong Kong Polytechnic University, Kowloon, Hong Kong

\author{
Email address: \\ 16109165g@connect.polyu.hk
}

\section{To cite this article:}

Lin Zhong. DEM Modelling of Particle Movement During Seepage Failure. Science Journal of Chemistry. Vol. 9, No. 3, 2021 , pp. 62-67. doi: $10.11648 /$ j.sjc.20210903.11

Received: February 4, 2021; Accepted: February 15, 2021; Published: May 14, 2021

\begin{abstract}
The Discrete Element Method (DEM) is a calculation method by which a particle flow at a single particle occurs. It is widely used in many fields such as coastal engineering, mining and civil engineering. Specifically, when small particles are taken away by water, pores will increase and collapse due to the soil's own weight, which will cause other parts of the soil to deform. Due to the continuous development of piping, small soil particles are taken away from the entrance, so the entrance is constantly expanding. Once the upper layer of soil loses the support below, the upper layer of particles will collapse under infiltration. Therefore, as the above phenomenon continues to develop, the amount and flow of water will increase. At the same time, this will increase the rate of seepage destruction. In fact, the law of particle motion includes the growth, aggregation, diffusion and crystallization of particles under different conditions, which can be applied to other fields, such as the research of material surface roughness in textile science, the crystal origin and potential self-rotation in electronic engineering, etc. The research of this paper can provide a further theoretical basis and framework for the above direction, and pave the way for the majority of scholars to carry out the follow-up research work. In this article, EDEM (Electronic Discrete Element Method) modeling is used to simulate the movement of particles during the slip.
\end{abstract}

Keywords: Slip, Seepage, DEM, EDEM, Particle, Soil

\section{Introduction}

DEM is a useful tool for successfully designing, optimizing or simply analyzing systems and equipment Granular materials where inter-particle forces play a key role in many applications. In the daily life, the destruction of the slip caused some serious geological disasters, such as floods [1]. S. Deverel et al. told us that the deformation and movement of particles are very important to control the destruction of cracks [2]. Therefore, particle motion is a significant area to be studied. In 2003, J. Santamarina mentioned that the balance of power can determine different behaviors in the soil. In 2015, Sato, M. and Kuwano, R. found that a small amount of internal erosion can progress without significant collapse, but there is a risk of deterioration of the stratum structure. In 2016, D. Ma et al. have found that particles can be transferred inside the soil. In 2017, Y. Liang and others mentioned that the particle erosion rate plays an important role in the development of piping. In 2010, Yang et al. found the mechanical effect of particle breakage is very important for some geotechnical engineering problems, such as side friction of driven pile, durability of railway ballast and irreversible deformation caused by disintegration. According to Coop et al. (2004), these and other applications urge people to study the mechanical consequences of particle breakage through experiments, incorporate particle breakage into particle constitutive model. In the same year, Cheng et al. have used DEM to simulate particle breakage. Two alternative methods can be used to simulate particle breakage with DEM: using new and smaller fragments to replace broken particles; Or use bonded agglomerates. In 2008, Bolton et al. thought about one point: although the latter is very helpful for understanding the micromechanics of individual grains, the latter will become an impractical tool if it is intended to model a wider range of problems. In 2020, X. Jiang et al. mentioned that the erosion of individual soil particles increases the possibility of leakage. Therefore, understanding the particle movement during infiltration becomes crucial.

In this paper, EDEM software is used to simulate the 
particles in the seepage process.

\section{Literature Review}

To start the DEM simulation, a connection model must be established and implemented with corresponding input parameters. The contact model describes the behavior of elements under what conditions they contact each other. The most commonly used contact models include: Hertz Mindlin and JKR (Johnson-Kendall-Roberts) cohesion model [3], linear cohesion (Romaní Fernández and Nirschl, 2013). Hertz-Mindlin is used for EDEM due to its accurate and effective force calculation. It explains the area of influence of the contact of two elastomers. In this model: the normal force component is based on the Hertz contact theory [4], and the tangential force model is based on the work of Mindlin-Deresiewicz (Mindlin, 1989; Mindlin and Deresiewicz, 1989). Hertz-Mindlin with the JKR cohesive model is a cohesive contact model that also takes into account the influence of the Van de Waals force in the contact area, and allows users to model strong bonding systems, such as dry materials.

The number of publications in this field is growing rapidly, especially due to the development of new technologies such as shale gas and oil exploration and development, underground energy, carbon dioxide and water resources exploration $[5,6]$.

At present, the main analysis methods are numerical calculation and numerical simulation. With the development of computing power and small processes, models and methods such as DEM and LBM have been applied [7-9]. At the same time, these methods also begin to involve particle flow in porous media. An important DEM was initially developed by cundall and Strack [10].

In addition, the method is extended to the extended discrete element method, considering the coupling of thermodynamics [11]. For the particle system in fluid, there are four research problems: particle particle interaction [12, 13], particle fluid interaction [14, 15], particle wall interaction and particle accumulation [16].

The particles will be divided into different parts, each of which can be set at different diameters. Therefore, the composite plugging effect of different particle sizes can be simulated. Each simulation is carried out at a given concentration, and it is obvious that when the particle concentration is low, it will not bridge. With the increase of particle concentration, the particle growth effect gradually appears, while other parameters remain unchanged in the calculation process. Finally, the particle size and fluid pressure are visualized and digitized at the outlet of the model. In this way, the movement effect can be tested. At the same time, the particle trajectory, model pressure and fluid velocity can be observed by simulation.

\section{Materials and Methods}

Part I

The schematic diagram of seepage in dike facilities is shown in Figure 1.

Piping of Embankment Foundation

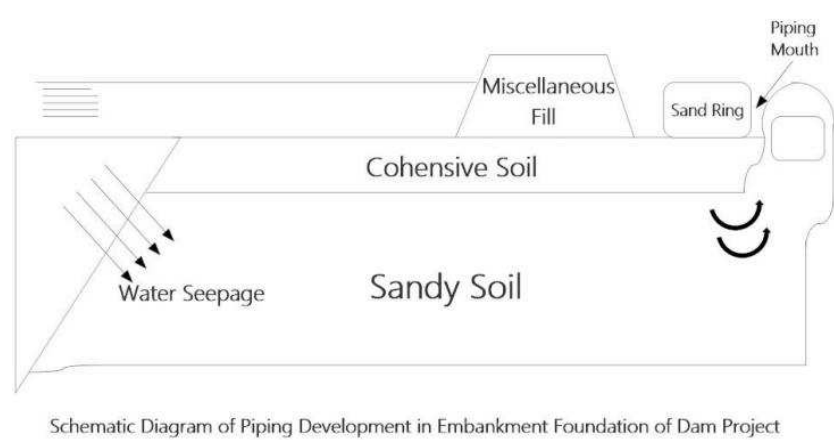

Figure 1. Schematic diagram of seepage.

In the second part, the process of building a simulation model will be shown step by step.

Part II

First, let's first construct the granular material. Here, the basic parameters are as follows: Poisson's ratio is 0.3 , density is 1500 , and shear modulus is $1 \mathrm{e}+10 \mathrm{~Pa}$. Because of the contact between the granular materials, the contact parameters need to be set in advance: the expansion recovery coefficient is 0.3 , the static friction is 0.5 , and the rolling friction is 0.01 . This is the basic parameter of granular materials.

Follow the procedure adopted by Tomasetta et al. (Tomasetta et al., 2014), for each consolidation condition, the tensile strength $\sigma \mathrm{t}$ can be calculated as the following:

$$
\sigma_{t}=\frac{C}{\tan \phi}
$$

Where $\sigma \mathrm{t}$ is the tensile strength, $\mathrm{C}$ is the cohesive force, and $\varphi$ is the angle of internal friction. The correlation between Van der Waals force and $\sigma \mathrm{t}$ is as follows:

$$
F_{v d W, 1}=\sigma_{t} d_{s v}^{2} \frac{\varepsilon}{1-\varepsilon}
$$

Where $d_{\mathrm{sv}}$ is the Sauter average diameter of the particles and $\varepsilon$ is the porosity of the bulk solid. Formula (2) follows the methods of Rumpf (Rumpf, 1970) and Molerus (Molerus, 1975), and combines the tensile strength with the binary Force between particles. Equations (1) and (2) can be used to estimate the interparticle force based on the measured powder fluidity. Another way to theoretically estimate van der Waals force is:

$$
F_{v d W, 2}=\frac{A r}{12 z_{0}^{2}}
$$

Where $\mathrm{A}$ is the Hamaker constant, $\mathrm{r}$ is the average radius of curvature of the contact point, and $\mathrm{z}_{0}$ is the separation distance (Israelachvili, 2011).

Next, after setting the particle material, we need to add particles-this article uses a single spherical particle. Then, set 
the radius to $10 \mathrm{~mm}$. The purpose of building a particulate material model is to simulate the movement of soil particles in the soil. For simulating the seepage problem of a dam, a bonded contact model needs to be added. For particles, in addition to the physical radius, a contact radius is also required. Here, the contact radius is set to $12 \mathrm{~mm}$. Now, briefly discuss the bonding model. The contact radius in the bonding model does not directly participate in the force calculation, and the physical radius plays a major role in the model calculation. The function of the contact radius here is: for example, the contact radius is $12 \mathrm{~mm}$, that is, the contact distance between two particles (two spherical surfaces) is $4 \mathrm{~mm}$. When the contact radii of the two particles overlap, they will form a bond with the chemical bonds are similar, that is, they will stimulate the bonding model, forming bonding bonds between them, forming a glue-like effect, and bonding them together. In other words, the contact radius defines a range by which it can be judged when two contact particles form a bond. Generally speaking, the contact radius should not be too large, but not too small. If the contact radius is set too large, it is easy to form bonds between particles that are far apart during the simulation.

In this case, the reliability of the simulation is not very high, which will lead to erroneous simulation results. If the contact radius is relatively small, the formed bond will be more brittle and easier to break, showing very fragile material properties, which will also lead to low reliability simulation results. The particle size distribution uniformly uses a fixed size.

The next step is equipment materials. The Poisson's ratio is 0.25 , the density is 7500 , and the shear modulus is $1 \mathrm{e}+10 \mathrm{~Pa}$. After setting, it is the "interaction" contact parameter. This type of parameter is very important for the influence of particle-particle and particle contact geometry: the expansion recovery coefficient is 0.2 , and the default values of static friction and rolling friction are 0.5 and 0.01 .

Next, add a box. The function of this box is to allow particles to move in this box for subsequent particle analysis. The size of this box is $500 \mathrm{X} 1000 \mathrm{X} 120$, and the unit is $\mathrm{mm}$. Since the particle object will definitely be affected by downward gravity, it is necessary to add a linear translation motion mode here: the start time of the motion is $0.4 \mathrm{~s}$, the end time is $0.5 \mathrm{~s}$, the initial speed is $0.65 \mathrm{~m} / \mathrm{s}$, and the particles move downward. Since this box is a closed body, it can be used as a pellet factory that allows pellets to move. Then, the pellet factory became a dynamic mode at this time. Set the total mass of the particles to $15 \mathrm{~kg}$ and generate $50 \mathrm{~kg}$ of particles per second.

Next, set the simulation time of contact and the parameters related to bonding. Since the end time of particle motion is set to $0.5 \mathrm{~s}$, the simulation start time of contact should be $0.6 \mathrm{~s}$. If you want to achieve the effect of "no matter how the particles change, the bond will not break", the limit normal stress and limit tangential stress should be as large as possible, so the normal stress and tangential stress here are both $1 \mathrm{e}+10 \mathrm{~Pa}$. Therefore, there are two factors that affect whether the bond between particles can be broken: the first is the contact radius-if the distance between two particles exceeds a certain range, the bond between them will fail; the other affects The factors are "limit normal stress" and "limit tangential stress"-if the bond is not broken, these two values should be as large as possible within a reasonable range, because whether the bond is healthy or not depends on These two parameters. On the other hand, for "normal stiffness per unit area" and "tangential stiffness per unit area", these two parameters determine the characteristics of the particle-for example, if these two parameters are too large, the difference between them may be greater Fragile, so by default, these two parameters are $1 \mathrm{e}+08$.

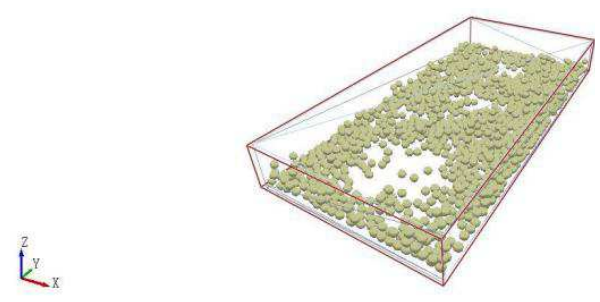

Figure 2. Preliminary simulation of particle model/

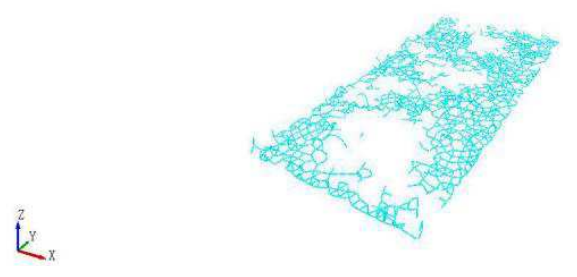

Figure 3. Discrete simulation of particle

Next, add a cylinder to match the shape when water seeps. The calculation domain needs to be adjusted. The $\mathrm{x}$-axis and $y$-axis remain unchanged, and the z-direction should be adjusted larger (to ensure that the range of falling particles is still within the entire computational domain).

As shown in Figure 4 and Figure 5.

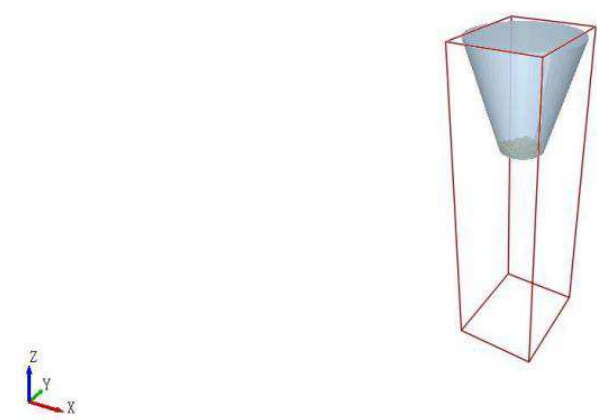

Figure 4. Full simulation of particle and contact force.

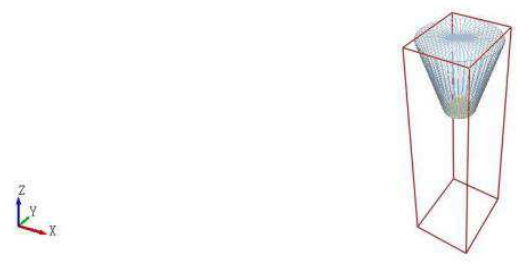

Figure 5. Complete simulation in mesh form. 
After completing the above steps, simulation and post-processing can be performed.

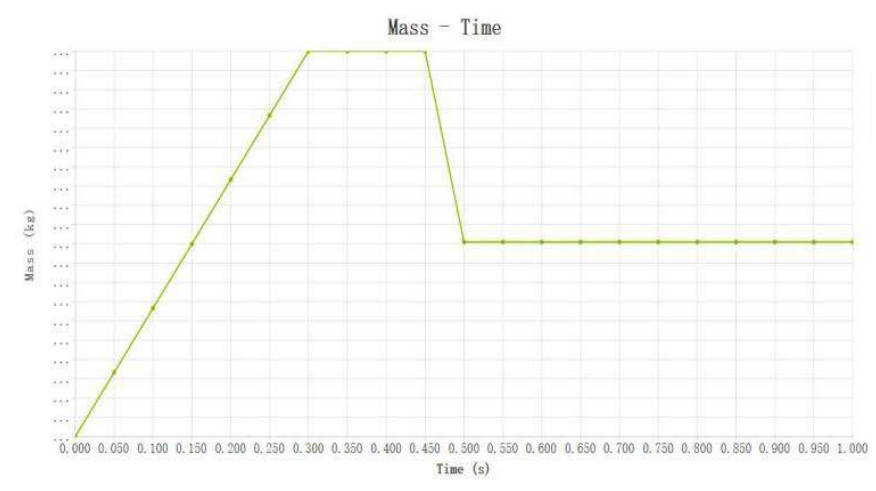

(a)

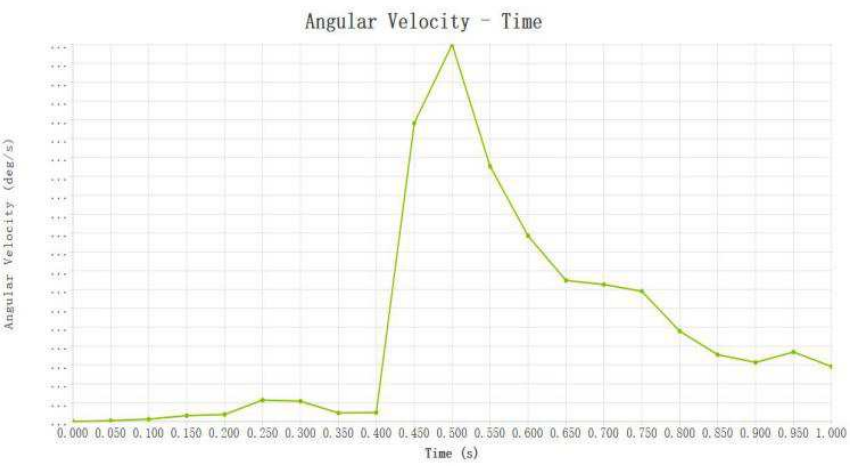

(b)

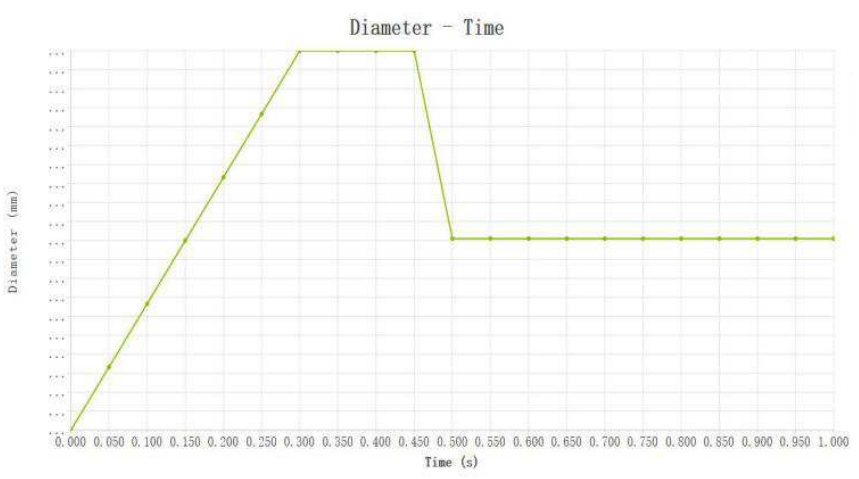

(c)

Figure 6. (a-c): Parameter variation trend during simulation.

Time: $0.0100016 \mathrm{~s}$

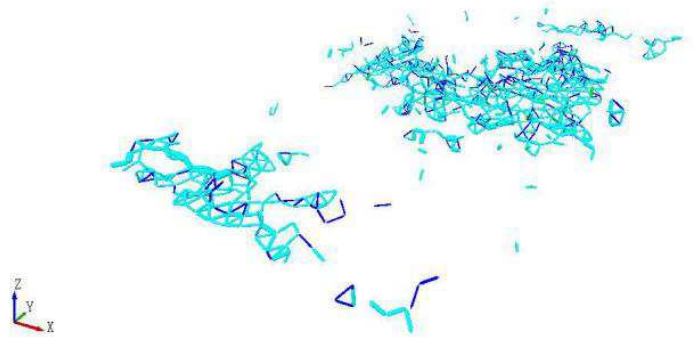

(a)
Time: $0.3 \mathrm{~s}$

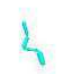

$k^{2}$

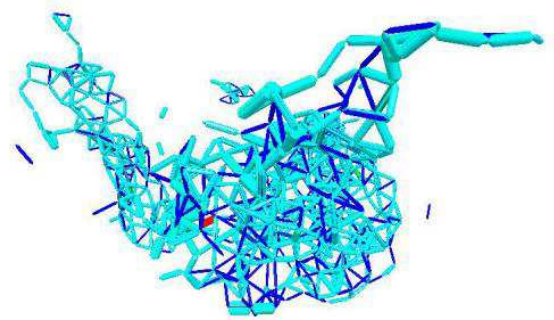

(b)

Time: $0.35 \mathrm{~s}$

ว

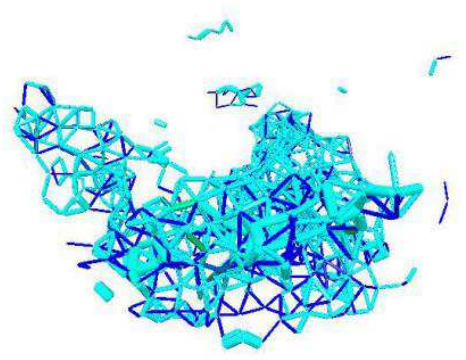

(c)

Time: $0.4 \mathrm{~s}$

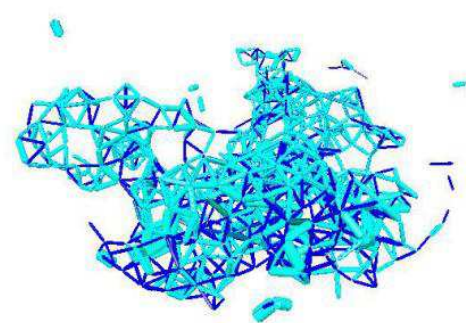

(d)

Time: $0.45 \mathrm{~s}$

,

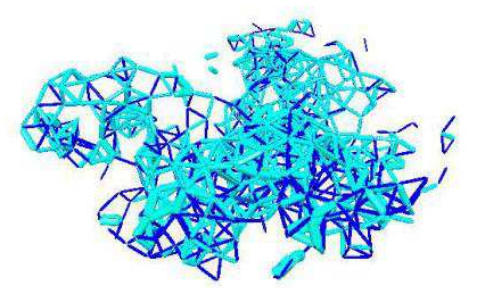

(e) 
Time: $0.5 \mathrm{~s}$

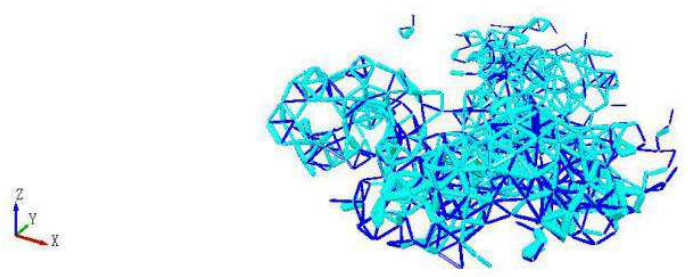

(f)

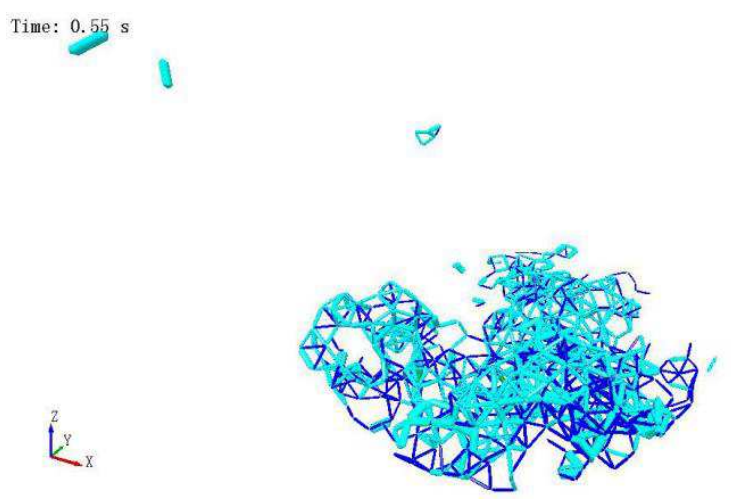

(g)

Time: $0.6 \mathrm{~s}$

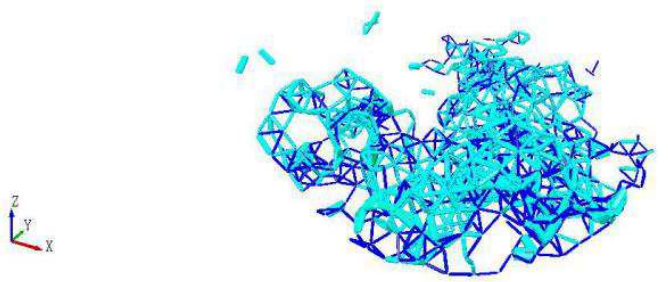

(h)

Time: $2 \mathrm{~s}$

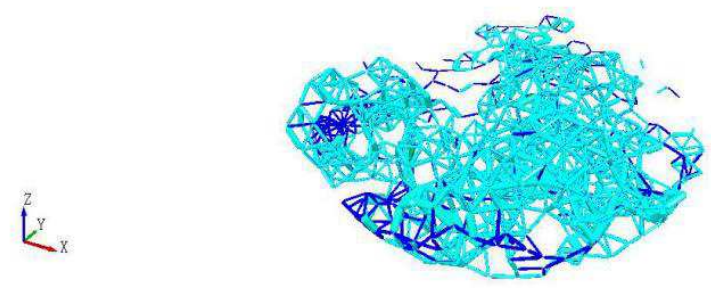

(i)

Figure 7. (a-i): Particle simulation during different time interval.

\section{Results and Discussion}

As shown in Figure 6, with the occurrence of seepage, the mass of soil particles becomes larger and larger, reaching the maximum value at $0.3 \mathrm{~s}$, and then remains unchanged until $0.45 \mathrm{~s}$. Starting from $0.4 \mathrm{~s}$, the particle quality began to decrease until 0.5 seconds. Starting from $0.5 \mathrm{~s}$, the particle mass remains unchanged. At the same time, the angular velocity of the particles also changed suddenly at $0.4 \mathrm{~s}$, increasing exponentially, slowing down at $0.45 \mathrm{~s}$, and reaching the peak angular velocity of $0.5 \mathrm{~s}$. Starting from $0.5 \mathrm{~s}$, the angular velocity begins to decrease until the end of the simulation. It is worth noting that even at the end of the simulation, the angular velocity of the particles is still greater than 0 , which means that the particles are still moving. On the other hand, the change in particle size is the same as the change in particle mass.

Figure 7 shows the change trend of the particle force size distribution over time in the seepage process. Here, sea blue indicates that the force received by the particle is the average of the force of the entire particle group, dark blue indicates that the force received by the particle is the minimum of the force of the entire particle group, and red represents a certain force. The force that each particle bears belongs to the largest force in the entire particle group. Initially, the particles are in a dispersed state, and there is no very close relationship between them (Figure 7a). When the time is from $0.01 \mathrm{~s}$ to $0.3 \mathrm{~s}$, the particles develop toward the aggregate state. At this time, more and more dark blue particles were formed. Not only that, but red particles will also appear, which means that at least one particle must bear the greatest force to form the appearance of the entire particle group toward the direction of aggregation (Figure 7b). As time passed, the dark blue particles and the sea blue particles gathered more and more between each other. When the network structure between the two slowly develops along the overlapping direction, the angular velocity and total mass of the stable particles also increase (Figure 6a-b, Figure 7c-i)

\section{Conclusion}

This article first shows the distribution of various soil components in the dam seepage problem and the schematic figure of the whole process. Then explain step by step how to build particle materials, particle groups and initial particle models. Then, the geometric body formed at the beginning of the seepage flow is regarded as the contact body and analyzed as the contact body of the particle model. In the end, it was found that within a few seconds, as long as the particles received the maximum force, the shape of the grid between the particles would change from dispersion to aggregation, until finally overlap. In general, the abstract particle motion patterns in the soil are displayed in the form of simulation modeling, which provides a basis for subsequent research. 


\section{References}

[1] Jaffe, David A., and Brett F. Sanders. 2001. "Engineered Levee Breaches for Flood Mitigation.” Journal of Hydraulic Engineering.

https://doi.org/10.1061/(asce)0733-9429(2001)127:6(471).

[2] Deverel, Steven J., Sandra Bachand, Scott J. Brandenberg, Cathleen E. Jones, Jonathan P. Stewart, and Paolo Zimmaro. 2016. "Factors and Processes Affecting Delta Levee System Vulnerability." San Francisco Estuary and Watershed Science. https://doi.org/10.15447/sfews.2016v14iss4art3.

[3] Baron, O., A., Rame', E., Wilkinson, A., 2009, DEM simulation of a schulze ring shear tester, in: AIP Conference Proceedings. pp. 409-412.

[4] Hertz, H., 1881, U'ber die Beru'hrung fester elastischer Ko'rper, Journal fu'r die reine und angewandte Mathematick, 92, 156-171.

[5] Lebeau M, Konrad JM. A new capillary and thin film flow model for predicting the hydraulic conductivity of unsaturated porous media. Water Resour Res 2010: 46 (12).

[6] Firouzi M, Alnoaimi K, Kovscek A, Wilcox J. Klinkenberg effect on predicting and measuring helium permeability in gas shales. Int J Coal Geol 2014; 123: 62-8.

[7] Zarghami A, Maghrebi MJ, Ghasemi J, Ubertini S. Lattice Boltzmann finite volume formulation with improved stability. Commun Comput Phys 2012; 12 (1): 42-64.

[8] Robinson M, Ramaioli M, Luding S. Fluid-particle flow simulations using two-way-coupled mesoscale SPH-DEM and validation. Int J Multiph Flow 2014; 59: 121-34.

[9] Zarghami A, Biscarini C, Succi S, Ubertini S. Hydrodynamics in porous media: a finite volume lattice Boltzmann study. J Sci Comput 2014; 59 (1): 80-103.

[10] Cundall PA, Strack OD. A discrete numerical model for granular assemblies. Geotechnique 1979; 29 (1): 47-65.

[11] Ning DZ, Shi J, Zou QP, Teng B. Investigation of hydrodynamic performance of an OWC (oscillating water column) wave energy device using a fully nonlinear HOBEM (higher-order boundary element method). Energy 2015; 83: 177-88.

[12] Heydarabadi H, Abdolzadeh M, Lari K. Simulation of airflow and particle deposition settled over a tilted Photovoltaic module. Energy 2017; 139: 1016-29.

[13] Deen N, Annaland MVS, Van der Hoef MA, Kuipers J. Review of discrete particle modelling of fluidized beds. Chem Eng Sci 2007; 62 (1-2): 28-44.

[14] Tsuji T, Yabumoto K, Tanaka T. Spontaneous structures in three-dimensional bubbling gas-fluidized bed by parallel DEM-CFD coupling simulaion. Powder Technol 2008; 184 (2) 132-40.

[15] Cleary PW. Industrial particle flow modelling using discrete element method. Eng Comput 2009; 26(6): 698-743.

[16] Abichou T, Benson CH, Edil TB. Network model for hydraulic conductivity of sand-bentonite mixtures. Can Geotech J 2004; 41 (4): 698-712.

[17] Santamarina, J. Carlos. 2003. "Soil Behavior at the Microscale: Particle Forces.” In. https://doi.org/10.1061/40659(2003)2.
[18] Sato, M., and R. Kuwano. 2015. "Effect of Internal Erosion on Deformation Characteristics in the Triaxial Compression Test." In Scour and Erosion - Proceedings of the 7th International Conference on Scour and Erosion, ICSE 2014. https://doi.org/10.1201/b17703-28.

[19] Chirone, R., Raganati, F., Ammendola, P., Barletta, D., Lettieri, P., Poletto, M., 2018, A comparison between interparticle forces estimated with direct powder shear testing and with sound assisted fluidization, Powder Technology, 323, 1-7. https://doi.org/10.1016/j.powtec.2017.09.038

[20] Gröger, T., Tüzün, U., Heyes, D. M., 2003, Modelling and measuring of cohesion in wet granular materials, Powder Technology, 133 , https://doi.org/10.1016/S0032-5910(03)00093-7

[21] Israelachvili, J., 2011, Intermolecular and Surface Forces, Intermolecular and Surface Forces. https://doi.org/10.1016/C2009-0-21560-1

[22] Mindlin, R. D., Deresiewicz, H., 1989, Elastic Spheres in Contact Under Varying Oblique Forces, in: The Collected Papers of Raymond D. Mindlin Volume I. pp. 269-286. https://doi.org/10.1007/978-1-4613-8865-4_35

[23] Obermayr, M., Vrettos, C., Eberhard, P., Däuwel, T., 2014, A discrete element model and its experimental validation for the prediction of draft forces in cohesive soil, Journal of Terramechanics, 53, 93104.https://doi.org/10.1016/j.jterra.2014.04.003

[24] Zhang, R., Li, J., 2006, Simulation on mechanical behavior of cohesive soil by Distinct Element Method, in:Journal of Terramechanics. $303-316$. https://doi.org/10.1016/j.jterra.2005.05.006

[25] Ma, Dan, Xiexing Miao, Haibo Bai, Hai Pu, Zhanqing Chen, Jiangfeng Liu, Yanhua Huang, Guimin Zhang, and Qi Zhang. 2016. "Impact of Particle Transfer on Flow Properties of Crushed Mudstones." Environmental Earth Sciences. https://doi.org/10.1007/s12665-016-5382-2.

[26] Liang, Y., C. Zeng, J. J. Wang, M. W. Liu, T. C. Jim Yeh, and Y. Y. Zha. 2017. "Constant Gradient Erosion Apparatus for Appraisal of Piping Behavior in Upward Seepage Flow." Geotechnical Testing Journal https://doi.org/10.1520/GTJ20150282.

[27] Jiang, Xiangang, Anders Wörman, Pengshuai Chen, Qin Huang, and Huayong Chen. 2020. "Mechanism of the Progressive Failure of Non-Cohesive Natural Dam Slopes." Geomorphology. https://doi.org/10.1016/j.geomorph.2020.107198.

[28] Yang, Bin, Tianhong Yang, Zenghe Xu, Honglei Liu, Xin Yang, and Wenhao Shi. 2019. "Impact of Particle-Size Distribution on Flow Properties of a Packed Column." Journal of Hydrologic Engineering. https://doi.org/10.1061/(asce)he.1943-5584.0001735.

[29] Fox, G. A., M. L. Chu-Agor, R. M. Cancienne, and G. V. Wilson. 2008. "Seepage Erosion Mechanisms of Bank Collapse: Three-Dimensional Seepage Particle Mobilization and Undercutting." In World Environmental and Water Resources Congress 2008: Ahupua'a - Proceedings of the World Environmental and Water Resources Congress 2008. https://doi.org/10.1061/40976(316)292.

[30] Okeke, Austin Chukwueloka Udechukwu, and Fawu Wang. 2016. "Critical Hydraulic Gradients for Seepage-Induced Failure of Landslide Dams." Geoenvironmental Disasters. https://doi.org/10.1186/s40677-016-0043-z. 